\title{
Research on Coal Exploration Technology Based on Satellite Remote Sensing
}

\author{
Dong Xiao, ${ }^{1}$ Ba Tuan Le, ${ }^{1,2}$ Yachun Mao, ${ }^{3}$ Jinhong Jiang, ${ }^{1}$ Liang Song, ${ }^{3}$ and Shanjun Liu ${ }^{3}$ \\ ${ }^{1}$ Information Science \& Engineering School, Northeastern University, Shenyang 110004, China \\ ${ }^{2}$ Control Technology College, Le Quy Don Technical University, Hanoi 100000, Vietnam \\ ${ }^{3}$ College of Resources \& Civil Engineering, Northeastern University, Shenyang 110004, China \\ Correspondence should be addressed to Dong Xiao; xiaodong@ise.neu.edu.cn
}

Received 10 December 2015; Revised 6 April 2016; Accepted 18 April 2016

Academic Editor: Yu-Lung Lo

Copyright (C) 2016 Dong Xiao et al. This is an open access article distributed under the Creative Commons Attribution License, which permits unrestricted use, distribution, and reproduction in any medium, provided the original work is properly cited.

\begin{abstract}
Coal is the main source of energy. In China and Vietnam, coal resources are very rich, but the exploration level is relatively low. This is mainly caused by the complicated geological structure, the low efficiency, the related damage, and other bad situations. To this end, we need to make use of some advanced technologies to guarantee the resource exploration is implemented smoothly and orderly. Numerous studies show that remote sensing technology is an effective way in coal exploration and measurement. In this paper, we try to measure the distribution and reserves of open-air coal area through satellite imagery. The satellite picture of openair coal mining region in Quang Ninh Province of Vietnam was collected as the experimental data. Firstly, the ENVI software is used to eliminate satellite imagery spectral interference. Then, the image classification model is established by the improved ELM algorithm. Finally, the effectiveness of the improved ELM algorithm is verified by using MATLAB simulations. The results show that the accuracies of the testing set reach $96.5 \%$. And it reaches $83 \%$ of the image discernment precision compared with the same image from Google.
\end{abstract}

\section{Introduction}

Remote sensing is a method of acquisition, transmission, processing, and extraction of geographic information without contacting with the surface directly. Compared with the conventional methods, remote sensing technology has features such as "multipoint," "multiband," and "temporal". At the same time, it can collect the information of wavelengths that fall well outside the visible spectrum, which enlarges the range of observation. The evolution trajectory of an objective phenomenon in the time dimension can be provided by repeated observations through remote sensing. Over the past 20 years, remote sensing technology has been used successfully in the forestry, agriculture, geological survey, and marine ecology fields. Interestingly, remote sensing technology is also an indispensable research technique in resources and environment studies. For the purposes of exploring coal mines, remote sensing technology can access a wide range of coal mining area information despite of the limits in the geographical conditions. This technology has been found applicable in coal exploration and measurement. For instance, Guan [1] proposed that airborne remote sensing data can be applied to coal forecast by the experimental study of space remote sensing in Taiyuan Coal. Zou [2, 3] studied the relationship between annular image, gravity, and magnetic anomalies on the satellite images of Hunan Xuefeng Coal. They found coal in Hunan Cenozoic cap by the use of the annular image. Later, based on multiple remote sensing messages for Landsat data and SPOT-5 data and airborne remote sensing data and other information, Mularz [4] explored the Belchatow lignite opencast mining area comprehensively and extracted change data of mining region by fusion of SPOT- 5 panchromatic in different time and TM multispectral image eventually. Also based on hyperspectral remote sensing data, interfered radar data, and GPS positioning measurement techniques, Cloutis [5] 
detected the effect of environment caused by exploitation coal mine underground in Ruhrgebiet area of German. Liu et al. [6] found that the geological phenomena under the cover layer influence the soil layer or surface feature (such as vegetation) directly or indirectly via the study of TM image of Huaibei Coal, which can be used to find buried geological coal. Recently, Tan et al. [7] presented a new multiband, multipolarization, and multiangle method to analyze remote sensing data and explore the geology of deep-level coal information. So far, a large number of studies have shown that remote sensing technology is an effective way to search for coal resource.

Pankiewicz technique is often used to categorize variety of images in the area of remote sensing satellite imagery classification. Generally speaking, these categorized methods can be divided into unsupervised classification, supervised classification, and neural networks. The nearest neighbor algorithm, histogram method, and clustering method [8-10] are the most common unsupervised classification methods. The supervised classification methods mainly include the nearest neighbor algorithm, maximum likelihood estimation, and support vector machine [11-13]. Artificial neural network based satellite image classification methods can be roughly divided into the PNN (Probability Neural Network), BP (Backpropagation) neural networks, and SOM (SelfOrganized Feature Map) [14, 15].

As is known, the variety of remote sensors is increasing and the image resolution of remote sensing is enhancing. However, the appropriate treatment, development, and application of the analytical tools are lagging behind. The traditional classification algorithm for the satellite image classification is likely to cause large scale and local minimum problems. Consequently, the speed and classification accuracy is far away from the demand. Therefore, to develop an accurate and fast automatic classification method in satellite imaging has always been a hot topic in the field of remote sensing.

To this end, this paper introduces a new single hidden layer feedforward extreme learning machine (ELM) and applies the algorithm to construct classifier for remote sensing satellite image classification. This novel algorithm is able to approach any complicated continuous functions and to learn new things with fast training speed and high accuracy. On the other hand, remote sensing technology has been widely applied to mining areas since 1970 [16-21]. However, most of these methods are subjective and timeconsuming because the spectral characteristics are unknown. In order to improve the accuracy of classification, the same experiment has to be conducted repeatedly. Instead the spectral characteristics may provide more comprehensive information. Based on the satellite image-spectral characteristics, we propose a method to search and measure coal. First, we use the ENVI software to eliminate interference of spectral image data. Then, we propose an improved ELM algorithm to establish coal image classification model. Finally, we use coal image classification model to classify satellite images of the Quang Ninh coal region in Vietnam. Simulation results show that the proposed algorithm has merits over many traditional neural networks.
TABLE 1: Bands of Landsat-5 TM.

\begin{tabular}{lccc}
\hline Band number & Band & Spectrum range $(\mu \mathrm{m})$ & Resolution $(\mathrm{m})$ \\
\hline B1 & Blue & $0.45-0.52$ & 30 \\
B2 & Green & $0.52-0.60$ & 30 \\
B3 & Red & $0.63-0.69$ & 30 \\
B4 & Near IR & $0.76-0.90$ & 30 \\
B5 & SW IR & $1.55-1.75$ & 30 \\
B6 & LW IR & $10.40-12.5$ & 120 \\
B7 & SW IR & $2.08-2.35$ & 30 \\
\hline
\end{tabular}

\section{Remote Sensing Reflectance Spectrum Data Preprocessing}

First, we downloaded the remote sensing images of coal mining of Vietnam Quang Ninh Province from USGS remote sensing image database. Landsat $5 \mathrm{TM}$ is selected as the satellite species which is the fifth in the US Landsat series and is an optical Earth observation satellite. Its payloads are thematic mapper (TM) and multispectral imager (MSS). The image acquired by Landsat- 5 satellite has been widely used in remote sensing of resources. The TM is divided into seven bands. The parameters of each band are shown in Table 1 . Since the sixth band is in hot infrared wavelength range, all the other six band (1st-5th and 7th) spectrums are used in this paper.

The original satellite imagery is vulnerable to the interference of atmosphere and surface reflectance of light, leading to distortion of the reflectance spectrum and geometry. Thus reflectance spectrum data must be verified before usage. The ENVI software is powerful in processing remote sensing image developed by American Exelis Visual Information Solutions, including input/output image data, scaling, image enhancement, correction, orthorectification, mosaic, information extraction, and image classification. ENVI can obtain the necessary information from remote sensing images quickly, easily, and accurately. Therefore we use ENVI to eliminate the influence of the surface reflections of the atmosphere and light. We can obtain a more realistic surface reflectance and reflectivity, surface temperature, and other physical model parameters, which can approach the actual spectral characteristics of the ground substance much better. ENVI spectral calibration consists of two steps, radiometric calibration and atmospheric correction.

2.1. Radiometric Calibration. Radiometric calibration is a process of quantifying the value of voltage recorded by the sensor or converting digital (DN) into absolute radiance value (reflectivity). ENVI provides tools specifically for radiometric calibration of Landsat satellite images, which can convert the DN value of TM into the value of radiation brightness or apparent reflectivity of atmospheric.

We open the calibration band and start the Landsat calibration tools. As shown in Figure 1, relevant parameters are set. A file must be created after six bands have been calibrated if only one band can be calibrated at a time. 


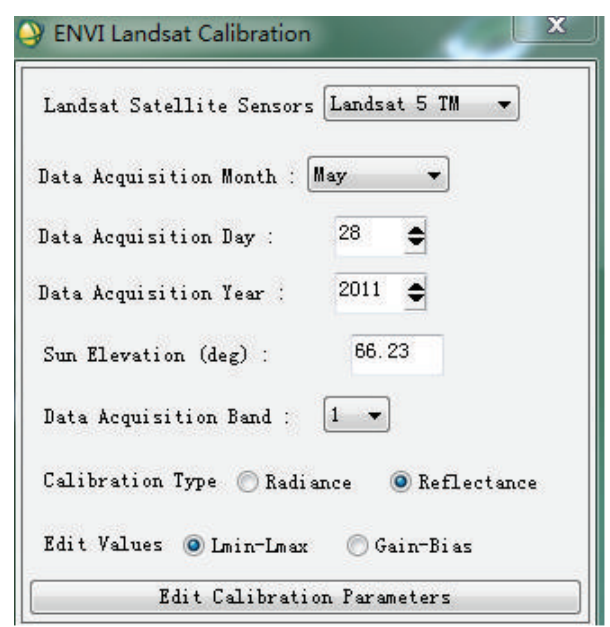

FIgURE 1: Landsat calibration tool box.

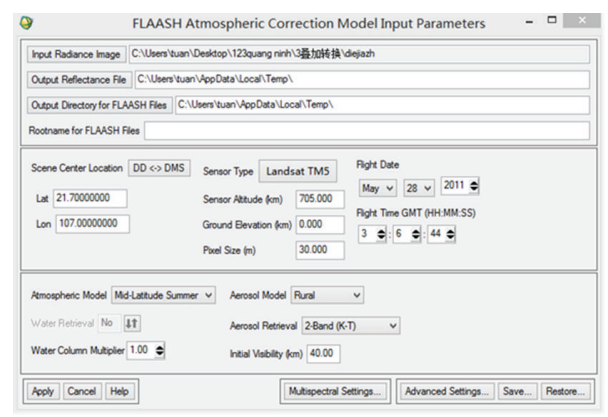

FIGURE 2: Input parameter of FLAASH.

2.2. Atmospheric Correction. ENVI contains a lot of atmospheric correction models. In this paper, atmosphere is corrected by MODTRAN model of Landsat FLAASH. Since TM data is common in BSQ format during atmospheric correction data by FLAASH in BIL format, the data conversion is needed before FLAASH atmospheric correction. After the data conversion, we start FLAASH atmospheric correction. FLAASH parameter settings are shown in Figure 2. The effects before and after atmospheric correction are shown in Figures 3 and 4 .

From reflectance spectrum of one point of the coal region in Figures 3 and 4, it can be seen that the coal of spectrum curve after correction is much closer to the real coal of spectrum curve.

2.3. Spectral Data Extraction. After the spectral calibration of satellite images, we extract the spectral data samples required for the experiment using the tie point method on image. The satellite images of coal mining area in Quang Ninh Province of Vietnam are chosen for spectral sampling. As shown in Figure 4, we make a right click at one point on mine image, select "Z Profile (spectrum)," click "File," and select "Save Plot As" to output the sample data in txt file format.

Due to the fact that the spectral data used in MATLAB cannot be directly extracted from satellite images, we need to read the spectral data of satellite images by ERDAS IMAGINE

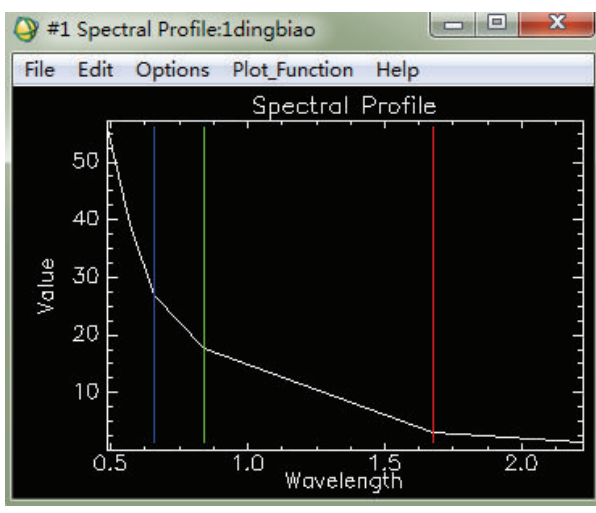

FIGURE 3: Spectrum before correction.

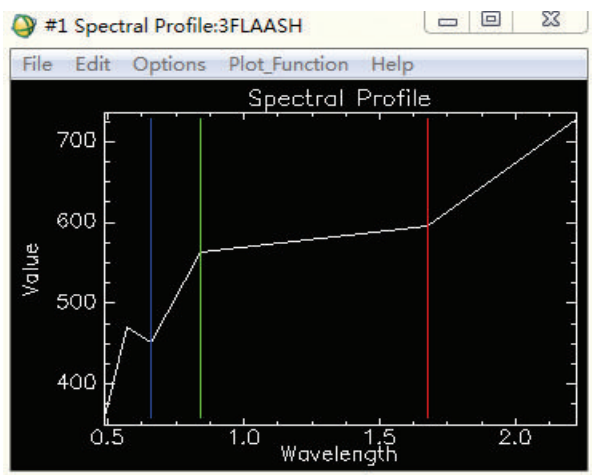

FIGURE 4: Spectrum after correction.

software which is developed by ERDAS corps in United States for remote sensing image processing. In ERDAS main menu, we click VIEWER, select the spectral data of the image in the popping dialog box, select To Be Read, select "Layer Info..." in the Utility, and click the pixel data. Then available MATLAB spectral data can be exported.

\section{Extreme Learning Machines}

The model of ELM proposed by Huang et al. [22] is constituted by input layer, single hidden layer, and output layer. The aim of ELM is to solve the above issues related to gradientbased algorithms. In ELM, input weights and single hidden layer biases are arbitrarily chosen without iterative adjust, and the only parameters to be learned in the training are the output weights which can be calculated by solving a single linear system [23]. Therefore, ELM is an efficient learning algorithm that has been widely applied in regression and multiclass classification.

We first define $N$ training samples, $\{X, T\}=\left\{x_{j}, t_{j}\right\}_{j=1}^{N}$, where $x_{j} \in \mathbf{R}_{p}$ and $t_{j} \in \mathbf{R}_{q}$ are the $j$ th input and target vectors, and the parameters $p$ and $q$ are the dimensions of input and target vector. To seek a regression function from the input to the target, one popular form is the standard single hidden layer feedforward network (SLFN) [24], where 
TABLE 2: Effect of accuracy of ELM neural network of different hidden layer nodes.

\begin{tabular}{lccc}
\hline Nodes of hidden layer & Training time $(\mathrm{s})$ & Average accuracy of training & Average accuracy of testing \\
\hline 10 & $0.0019 \mathrm{~s}$ & $70.2367 \%$ & $68.2119 \%$ \\
20 & $0.005 \mathrm{~s}$ & $79.1817 \%$ & $78.8929 \%$ \\
100 & $0.0381 \mathrm{~s}$ & $94.0133 \%$ & $90.9595 \%$ \\
200 & $0.1334 \mathrm{~s}$ & $96.615 \%$ & $92.5714 \%$ \\
250 & $0.2137 \mathrm{~s}$ & $97.2383 \%$ & $92.7524 \%$ \\
300 & $0.3050 \mathrm{~s}$ & $97.6183 \%$ & $92.9571 \%$ \\
350 & $0.4159 \mathrm{~s}$ & $97.9967 \%$ & $92.9905 \%$ \\
400 & $0.5419 \mathrm{~s}$ & $98.2 \%$ & $92.819 \%$ \\
500 & $0.8374 \mathrm{~s}$ & $98.505 \%$ & $92.481 \%$ \\
600 & $1.2403 \mathrm{~s}$ & $98.69 \%$ & $91.5976 \%$ \\
700 & $1.5686 \mathrm{~s}$ & $98.94 \%$ & $89.8357 \%$ \\
\hline
\end{tabular}

$n_{h}$ single hidden nodes fully connect the $p$ input nodes to the $q$ output nodes, which can be mathematically modeled as

$$
o_{j}=\sum_{i=1}^{n_{h}} \beta_{i} g\left(w_{i}^{T} x_{j}+b_{i}\right)=t_{j},
$$

where $o_{j} \in \mathbf{R}_{q}$ is the output vector of the $j$ th training sample, $w_{j} \in \mathbf{R}_{p}$ is the input weight vector connecting the input nodes to the $i$ th hidden node, $b_{i}$ is the bias of the $i$ th hidden node, and $g(\cdot)$ denotes hidden nodes nonlinear piecewise continuous activation functions.

The above $N$ equations can be written compactly as

$$
\mathbf{H} \beta=\mathbf{T},
$$

where the matrix $\mathbf{T}$ is target matrix,

$$
\begin{aligned}
& \mathbf{H}=\left[\begin{array}{ccc}
g\left(w_{1}^{T} x_{1}+b_{1}\right) & \cdots & g\left(w_{n_{h}}^{T} x_{1}+b_{n_{h}}\right) \\
\vdots & \cdots & \vdots \\
g\left(w_{1}^{T} x_{N}+b_{1}\right) & \cdots & g\left(w_{n_{h}}^{T} x_{N}+b_{n_{h}}\right)
\end{array}\right], \\
& \beta=\left[\begin{array}{c}
\beta_{1}^{T} \\
\vdots \\
\beta_{n_{h}}^{T}
\end{array}\right], \\
& \mathbf{T}=\left[\begin{array}{c}
t_{1}^{T} \\
\vdots \\
t_{N}^{T}
\end{array}\right] .
\end{aligned}
$$

The matrix $\mathbf{H}$ is the hidden layer output matrix, which can be randomly generated independent of the training data. $\beta=\left[\beta_{1}, \beta_{2}, \ldots, \beta_{n_{h}}\right]^{T}\left(\beta_{i} \in \mathbf{R}_{q}\right)$ is the output weight matrix between the hidden nodes and the output nodes. Thus, training SLFNs simply amounts to getting the solution of a linear system (2) of the output weights $\beta[25,26]$.

According to the theoretical results in [27, 28], ELM aims to reach the smallest training error:

$$
\text { Minimize: }\|\mathbf{H} \beta-\mathbf{T}\| \text {. }
$$

A simple representation of the solution of (4) is given explicitly by Huang et al. [24] as

$$
\widehat{\beta}=\mathbf{H}^{+} \mathbf{T}
$$

where $\mathbf{H}^{+}$is the Moore-Penrose generalized inverse of the hidden layer output matrix $\mathbf{H}$.

\section{Classification Model of Coal Satellite Image Based on Improved ELM Algorithm}

ELM algorithm is used to model, simulate, and analyze the sampled data. There are 600 training samples (300 for coal, 300 for noncoal) and 420 test samples (200 for coal, 220 for noncoal). This environment of experimental operating is in Windows 7 version, Intel processor Pentium (R)E6700@3.20 GHz Dual-Core, 2 GB memory, GeForce NVIDIA 405, and 2014b MATLAB. The experiment was repeated 100 times, and the results were as follows.

4.1. Model of Basic ELM. Simulation results are shown in Table 2 and Figure 5. It is seen that the accuracy of the training set and the test set is about $70 \%$ in the case that the nodes of hidden layer are relatively short; the accuracy is high and stable while the nodes of hidden layer are in 300 range and it can reach $92.9 \%$; if the number of nodes in the hidden layer is improved again, the accuracy of the training set is improved, but the accuracy of the test set is decreased; and the fact that training time is short while nodes in the hidden layer are relatively small.

4.2. Model of Integrated ELM. In order to improve the test precision, this paper presents a new method to improve the ELM neural network. The design idea can be summarized as follows: if the weights and biases of the ELM neural network are randomly given, the results are different from each output. We integrate several ELM models and save every test set of each model. Then a final vote is made to determine the final result. This procedure can be named as "integrated ELM classification algorithm." In the experiment, the number of 
TABLE 3: The effect of accuracy based on different number of ELM model.

\begin{tabular}{lccc}
\hline Number of model & Training time (s) & Average accuracy of training set & Average accuracy of testing set \\
\hline 1 & $0.3050 \mathrm{~s}$ & $97.6183 \%$ & $92.9571 \%$ \\
5 & $1.5536 \mathrm{~s}$ & $98.555 \%$ & $94.5595 \%$ \\
11 & $3.4102 \mathrm{~s}$ & $98.79 \%$ & $95.5048 \%$ \\
21 & $6.5255 \mathrm{~s}$ & $98.8383 \%$ & $95.5167 \%$ \\
41 & $12.7578 \mathrm{~s}$ & $98.845 \%$ & $95.5548 \%$ \\
101 & $32.9162 \mathrm{~s}$ & $98.983 \%$ & $95.6243 \%$ \\
\hline
\end{tabular}

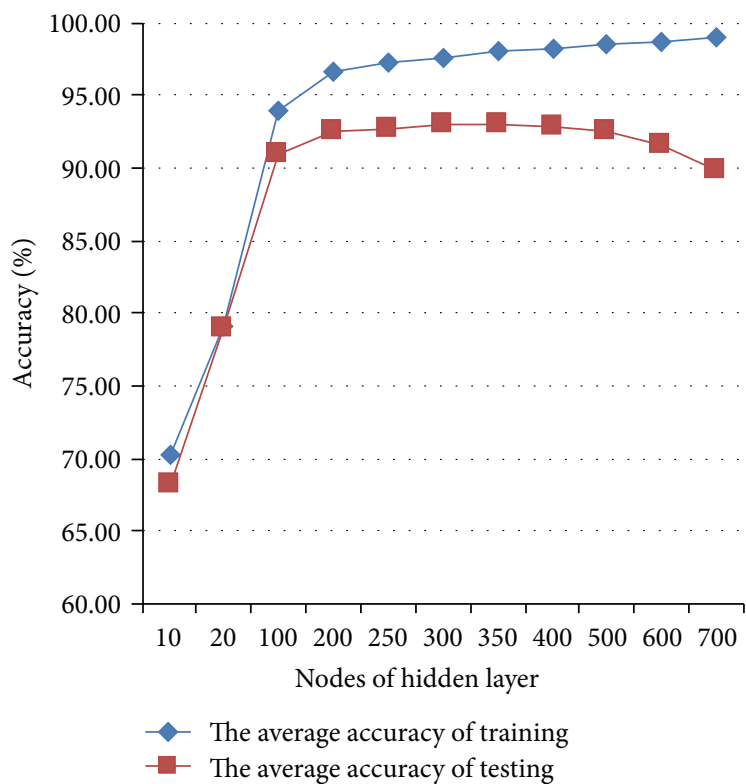

FIGURE 5: Effect of accuracy of training of different hidden layer nodes.

hidden layer nodes of ELM neural network is 300 . We change the number of ELM model, repeat the experiment 100 times, and average the results as the final result, which is shown in Table 3.

As shown in Table 3 and Figure 6, the accuracy of the test set by the integrated ELM model improves to more than $94 \%$; the higher the number of models, the higher the accuracy of the model, but the longer the training time; more precisely, the training time of the 11-model is about equal to the 5model, but the accuracy of the test is about $1.4 \%$ higher than that of the latter; the accuracy of test set of the 11-model is almost equal to the 101-model while the former is much faster than the latter. Relatively speaking, the 11-model may be the most reasonable parameter.

4.3. Model Based on Stochastic Optimization Integrated ELM. Because ELM weights and biases are random assigned, we further improve the integrated model. The design idea is summarized as follows: (i) Step one, train the individual model 100 times, compare the 100 results, and then save the

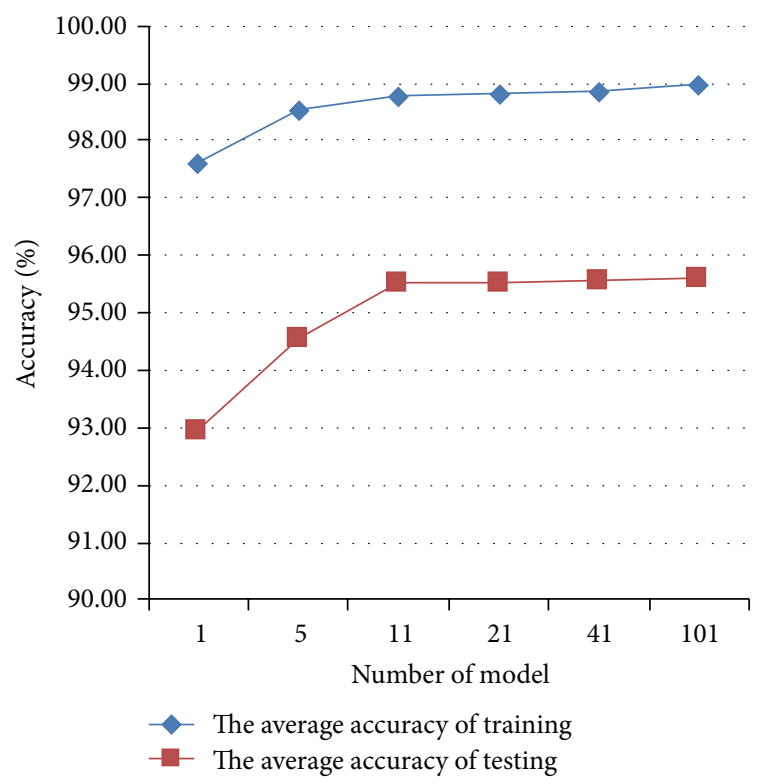

FIGURE 6: The effect of accuracy based on different number of ELM model.

weights and offset values of the best results of ELM algorithm; (ii) Step two, repeat Step one 11 times and find out the weights and biases of 11 best ELM neural network; (iii) Step three, we integrate the 11 individual ELM neural network models to vote. The model has two identifications. They are labeled as Class 1 and Class 2. If there are six or more results in Class 1 in the 11 results, we consider the result as Class 1. It is similar to Class 2. The method can be called stochastic optimization integrated ELM (XS-ELM) based on its features.

Table 4 and Figure 7, respectively, show the comparison results among the models of stochastic optimization integration ELM algorithm, basal ELM, integration ELM and traditional Vector Machine Support (SVM), and Backpropagation (BP).

From the numerical results, we conclude that stochastic optimization integrated XS-ELM has improved the accuracy of the test up to $96.55 \%$ which is $4 \%$ higher than the basic ELM and 1\% than integrated ELM model. The accuracy of the proposed XS-ELM algorithm is better than SVM and BP neural network method and even reaches $96.5 \%$. Its only drawback is that the training time is slightly longer. 
TABLE 4: The accuracy of the five methods.

\begin{tabular}{lccc}
\hline Method of ELM & Training time (s) & Average accuracy of training & Average accuracy of testing \\
\hline Basic ELM & $0.3050 \mathrm{~s}$ & $97.6183 \%$ & $92.9571 \%$ \\
Integrated ELM & $3.4102 \mathrm{~s}$ & $98.79 \%$ & $95.5048 \%$ \\
XS-ELM & $21.6991 \mathrm{~s}$ & $96.9629 \%$ & $96.5492 \%$ \\
SVM & $0.7510 \mathrm{~s}$ & $95.3745 \%$ & $95.4571 \%$ \\
BP & $5.8032 \mathrm{~s}$ & $98.83 \%$ & $94.29 \%$ \\
\hline
\end{tabular}

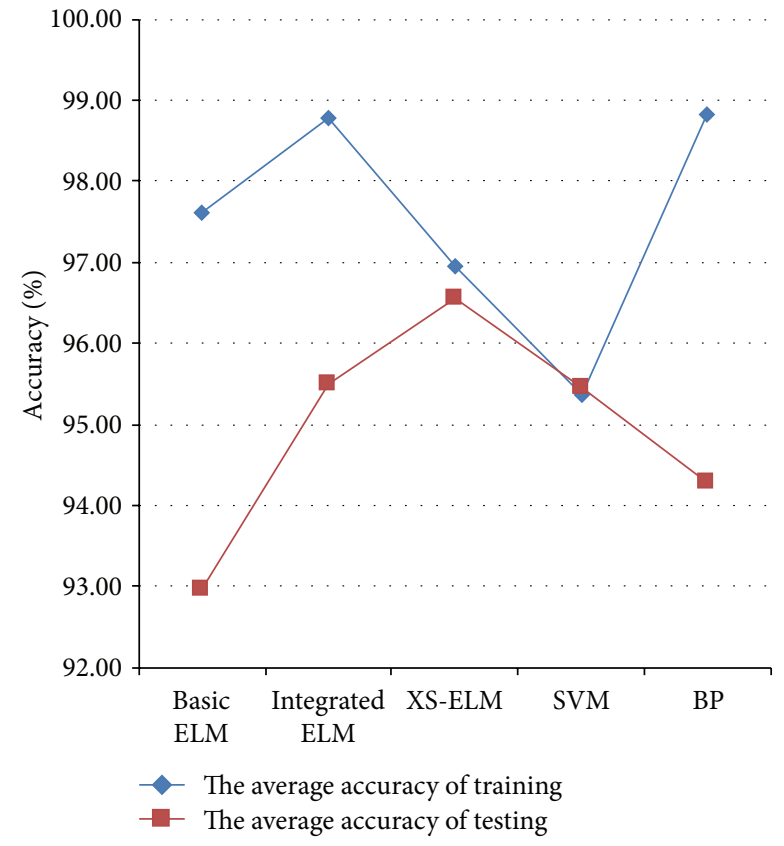

Figure 7: The accuracy of the five methods.

\section{Application of Improved ELM Algorithm in Remote Sensing for Coal}

The coal resource in Vietnam is rich in variety and good in quality. Vietnam is one of the most important countries for coal producing; the coal mines are mainly located in the northern region. Quang Ninh Province is known as the base of Vietnamese coal production, with a large open anthracite and a 100-year mining history till now. The area of coal reaches about 220 square kilometers with the volume about 3000 million tons in Quang Ninh Province known as one of the best anthracites in the world.

We preprocess the remote sensing image data by ENVI. Then, we use the coal identification model to identify the remote sensing image. The identification results are shown in Figures 8(a), 9(a), 10(a), 11(a), and 12(a). Finally, we compare the coal mine image and the image of Google map which is acquired from Google HD map in accurate latitude and longitude. Then we circle out the areas of coal mining and make a careful revision. At last we conclude that the accuracy of the image of coal mine area is $100 \%$. We compare the two images, select Google map image as the bottom layer, and
TABLE 5: The accuracy comparing the map and the identification of different ELM methods.

\begin{tabular}{ll}
\hline Method & Accuracy \\
\hline Basic ELM & $78.5679 \%$ \\
Integrated ELM & $81.3777 \%$ \\
XS-ELM & $83.0831 \%$ \\
SVM & $81.9066 \%$ \\
BP & $80.7854 \%$ \\
\hline
\end{tabular}

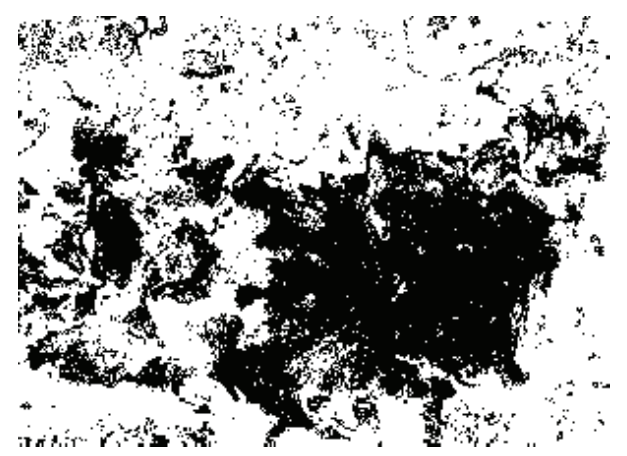

(a) Identification image of basic ELM

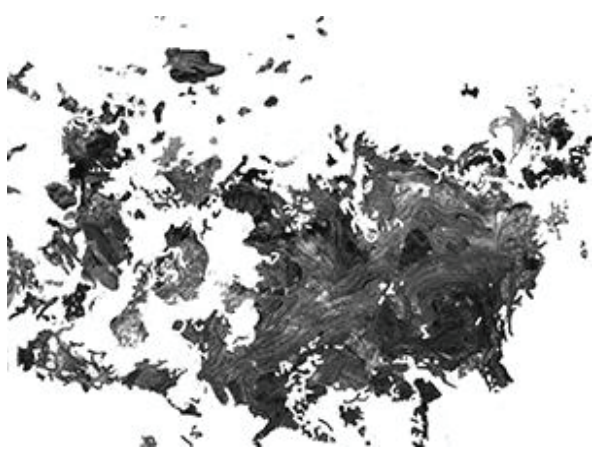

(b) Google map image of coal of Quang Ninh, Vietnam

FIGURE 8: Images of identification of basic ELM and Google map.

analyze the similarity of each pixel of the two images. The comparison results are shown in Figures 8-12 and Table 5.

Figures $8-12$ show the images of the coal mining area by the use of the basic ELM, integrated ELM, XS-ELM, SVM, and BP, respectively. We compare these images with Google maps by using MATLAB software. The accuracy of each method is shown in Table 5 (the basic ELM method is $78.5679 \%$; the integrated ELM method is $81.3777 \%$; the XS-ELM method is $83.0831 \%$; the SVM method is $81.9066 \%$; 


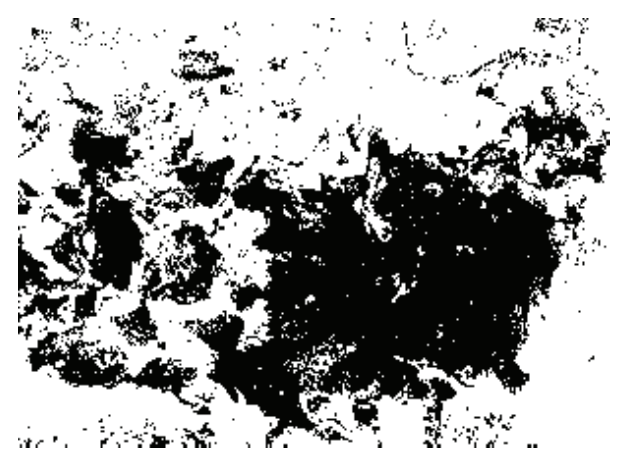

(a) Identification image of integrated ELM

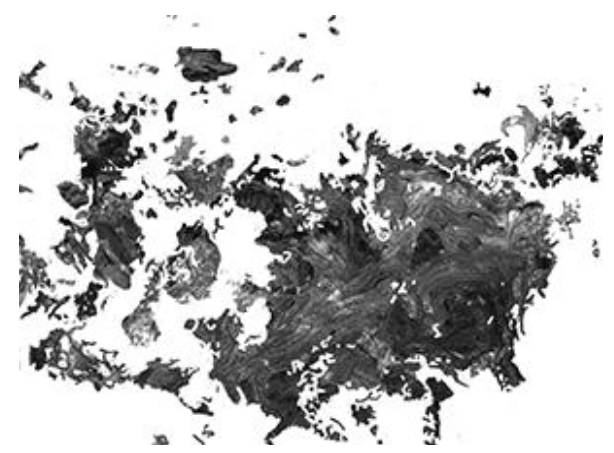

(b) Google map image of coal of Quang Ninh, Vietnam

Figure 9: Images of identification of integrated ELM and Google map.

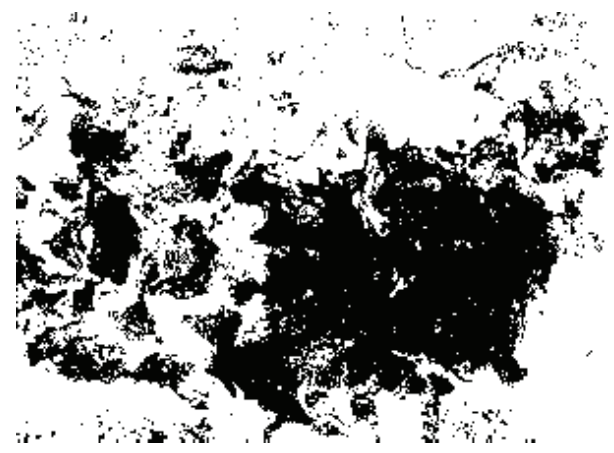

(a) Identification image of XS-ELM

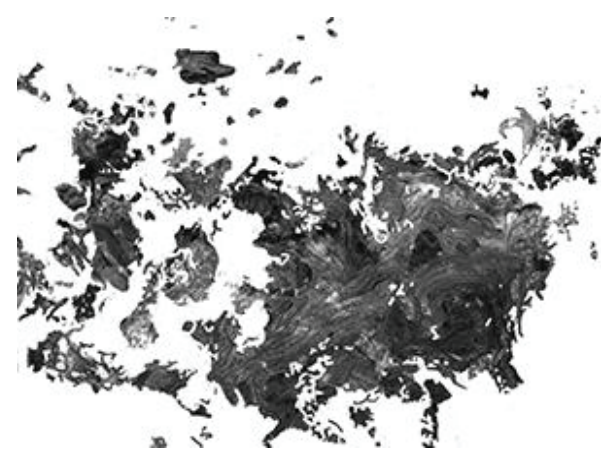

(b) Google map image of coal of Quang Ninh, Vietnam

FIGURE 10: Images of identification of XS-ELM and Google map.

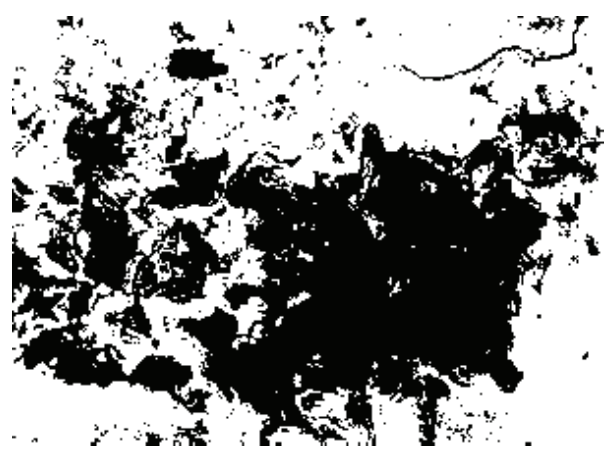

(a) Identification image of SVM

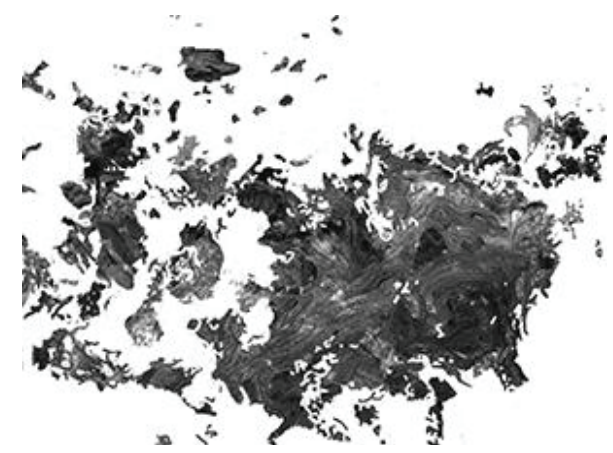

(b) Google map image of coal of Quang Ninh, Vietnam

FIGURE 11: Images of identification of SVM and Google map.

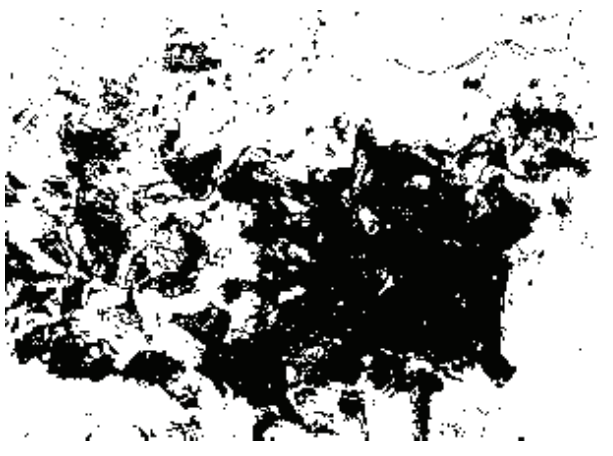

(a) Identification image of $\mathrm{BP}$

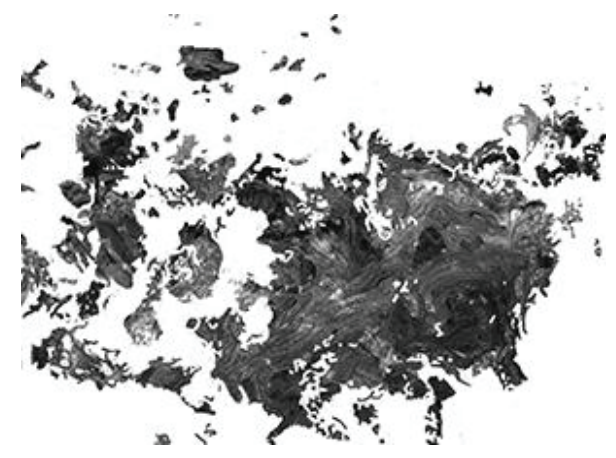

(b) Google map image of coal of Quang Ninh, Vietnam

FIGURE 12: Images of identification of BP and Google map. 
the BP method is $80.7854 \%$ ). Comparison results verify that the wide applicability of the proposed algorithm. The $83 \%$ accuracy of satellite image identification shows the effectiveness of this method. At the same time, the accuracy is higher than that of SVM and BP. The experimental results also show that the improved ELM algorithm can improve the accuracy of satellite image identification from $78.5 \%$ to $83 \%$.

\section{Conclusion}

Remote sensing technology is an important method to find coal and also plays an important role in the investigation of all kinds of resources and mineral exploration. It has a great number of advantages, wide field of view, strong macro, low cost, and high efficiency. We illustrate the practical examples of coal by using spectral information of remote sensing images. The method can be applied to the exploration, measure the distribution, and reserve of open-air coal mining area. In this paper, we use artificial intelligence system and remote sensing spectral characteristics to study coal resource exploration. Simulation results verify the merits of the proposed improved ELM algorithm over some other conventional methods. With the development of remote sensing technology, it is expected that the proposed method could be applied to other fields in future, such as geology, geography, science, and coal sedimentology.

\section{Competing Interests}

The authors declare that there are no competing interests regarding the publication of this paper.

\section{Acknowledgments}

This research is supported by National Natural Science Foundation of China (Grant numbers 41371437 and 61203214) and National Twelfth Five-Year Plan for Science and Technology Support (2015BAB15B01) of China.

\section{References}

[1] H. Y. Guan, "Application of remote sensing technology in coal field geology," Acta Geologica Sinica, no. 1, pp. 36-49, 1989.

[2] Y. Y. Zou, "Correlativity of the circular structure images with the gravity and magnetic anomalies and its implication for the future coal investigation," Coal Geology \& Exploration, vol. 20, no. 2, pp. 16-20, 1992.

[3] Y. Y. Zou, "Application and achievements of remote sensing to explore coal resource under Mesozoic-Cenozoic cap rock in Hunan Province," Coal Geology China, vol. 5, no. 3, pp. 58-60, 1993.

[4] S. C. Mularz, "Satellite and airborne remote sensing data for monitoring of an open-cast mine," IAPRS, vol. 32, no. 4, pp. 395402, 1998.

[5] E. A. Cloutis, "Hyperspectral geological remote sensing: evaluation of analytical techniques," International Journal of Remote Sensing, vol. 17, no. 12, pp. 2215-2242, 1996.

[6] Y. R. Liu, S. L. Wang, Y. Z. Ling, and S. N. Ning, "Technique of TM image information extraction and its geological effect in a buried coal field," Remote Sensing for Land and Resource, vol. 32, no. 3, pp. 29-33, 1997.

[7] K. L. Tan, Y. Q. Wan, X. F. Wang, S. X. Sun, and X. L. Chen, "The exploration method of coal resources based on remote sensing technology," Geology in China, vol. 39, no. 1, pp. 218-227, 2012.

[8] W. B. Sun, H. Y. Tang, H. X. Chen, and D. Wu, "Research of unsupervised change detection means based on clustering characteristic of 2-D histogram," in Proceedings of the 18th International Symposium on Intelligent Signal Processing and Communication Systems (ISPACS '10), pp. 1-4, IEEE, Chengdu, China, December 2010.

[9] A. A. K. Tahir, "Integrating artificial neural network and classical methods for unsupervised classification of optical remote sensing data," EURASIP Journal on Advances in Signal Processing, vol. 2012, article 165, 2012.

[10] N. Alajlan, N. Ammour, Y. Bazi, and H. Hichri, "A cluster ensemble method for robust unsupervised classification of VHR remote sensing images," in Proceedings of the IEEE International Geoscience and Remote Sensing Symposium (IGARSS '11), pp. 2896-2899, IEEE, Vancouver, Canada, July 2011.

[11] B. Demir, L. Minello, and L. Bruzzone, "Definition of effective training sets for supervised classification of remote sensing images by a novel cost-sensitive active learning method," IEEE Transactions on Geoscience and Remote Sensing, vol. 52, no. 2, pp. 1272-1284, 2014.

[12] C. Persello and L. Bruzzone, "Active learning for domain adaptation in the supervised classification of remote sensing images," IEEE Transactions on Geoscience and Remote Sensing, vol. 50, no. 11, pp. 4468-4483, 2012.

[13] P. M. Atkinson, "Spatially weighted supervised classification for remote sensing," International Journal of Applied Earth Observation and Geoinformation, vol. 5, no. 4, pp. 277-291, 2004.

[14] M. Şahin, "Modelling of air temperature using remote sensing and artificial neural network in Turkey," Advances in Space Research, vol. 50, no. 7, pp. 973-985, 2012.

[15] Y.-X. Gong, C. He, F. Yan et al., "Study on artificial neural network combined with multispectral remote sensing imagery for forest site evaluation," Spectroscopy and Spectral Analysis, vol. 33, no. 10, pp. 2815-2822, 2013.

[16] N. F. Parks, G. W. Peterson, and G. M. Baumer, "High resolution remote sensing of spatially and spectrally complex coal surface mores of central Pennsylvania: a comparison between simulated SPOT MSS and Landsat-5 Thematic Mapper," Photogrammetric Engineering and Remote Sensing, vol. 53, no. 4, pp. 415-420, 1987.

[17] A. Prakash and R. P. Gupta, "Land-use mapping and change detection in a coal mining area-a case study in the Jharia coalfield, India," International Journal of Remote Sensing, vol. 19, no. 3, pp. 391-410, 1998.

[18] H. L. Chen, G. Chen, J. L. Li, and G. P. Ding, "RS based ecological environmental dynamic monitoring in mining area," Resources Science, vol. 26, no. 5, pp. 132-138, 2004.

[19] W.-B. Wu, J. Yao, and T.-J. Kang, "Study on land use changes of the coal mining area based on TM image," Journal of Coal Science and Engineering, vol. 14, no. 2, pp. 287-290, 2008.

[20] L. Schroeter and C. Gläßer, "Analyses and monitoring of lignite mining lakes in Eastern Germany with spectral signatures of Landsat TM satellite data," International Journal of Coal Geology, vol. 86, no. 1, pp. 27-39, 2011.

[21] F. Tian, Y. J. Wang, R. Fensholt, K. Wang, L. Zhang, and Y. Huang, "Mapping and evaluation of NDVI trends from 
synthetic time series obtained by blending landsat and MODIS data around a coalfield on the loess plateau," Remote Sensing, vol. 5, no. 9, pp. 4255-4279, 2013.

[22] G.-B. Huang, Q.-Y. Zhu, and C.-K. Siew, "Extreme learning machine: a new learning scheme of feedforward neural networks," in Proceedings of the IEEE International Joint Conference on Neural Networks, vol. 2, pp. 985-990, IEEE, July 2004.

[23] G.-B. Huang, H. Zhou, X. Ding, and R. Zhang, "Extreme learning machine for regression and multiclass classification," IEEE Transactions on Systems, Man, and Cybernetics, Part B: Cybernetics, vol. 42, no. 2, pp. 513-529, 2012.

[24] G.-B. Huang, Q.-Y. Zhu, and C.-K. Siew, "Extreme learning machine: theory and applications," Neurocomputing, vol. 70, no. 1-3, pp. 489-501, 2006.

[25] G. Feng, G.-B. Huang, Q. Lin, and R. Gay, "Error minimized extreme learning machine with growth of hidden nodes and incremental learning," IEEE Transactions on Neural Networks, vol. 20, no. 8, pp. 1352-1357, 2009.

[26] G.-B. Huang, "What are extreme learning machines? Filling the Gap Between Frank Rosenblatt's Dream and John von Neumann's Puzzle," Cognitive Computation, vol. 7, no. 3, pp. 263-278, 2015.

[27] G.-B. Huang, "An insight into extreme learning machines: random neurons, random features and kernels," Cognitive Computation, vol. 6, no. 3, pp. 376-390, 2014.

[28] J. W. Cao, T. Chen, and J. Fan, "Landmark recognition with compact BoW histogram and ensemble ELM," Multimedia Tools and Applications, vol. 75, no. 5, pp. 2839-2857, 2016. 


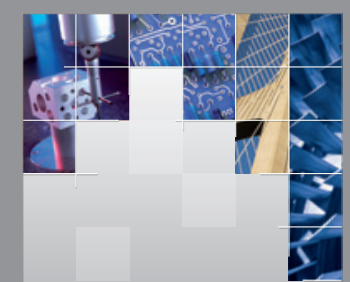

\section{Enfincering}
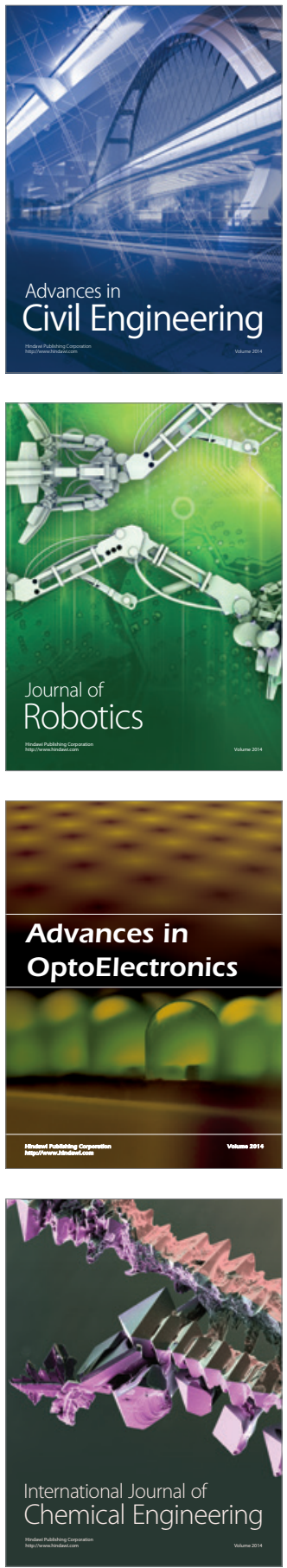

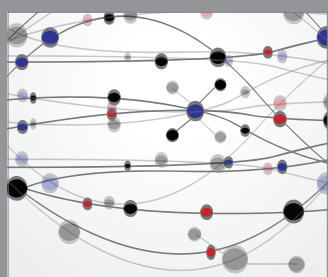

The Scientific World Journal

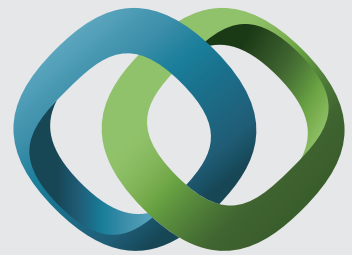

\section{Hindawi}

Submit your manuscripts at

http://www.hindawi.com
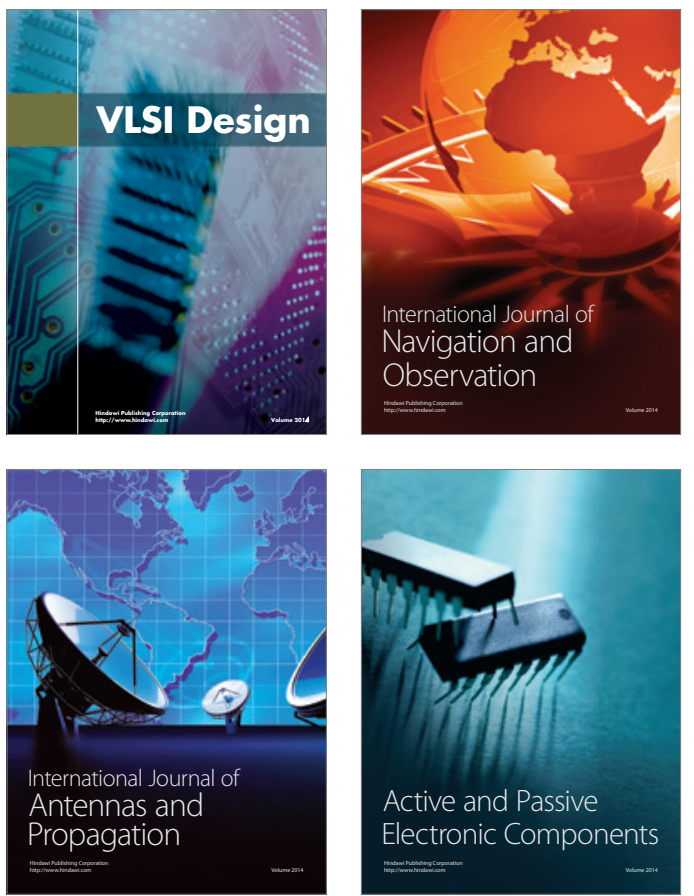
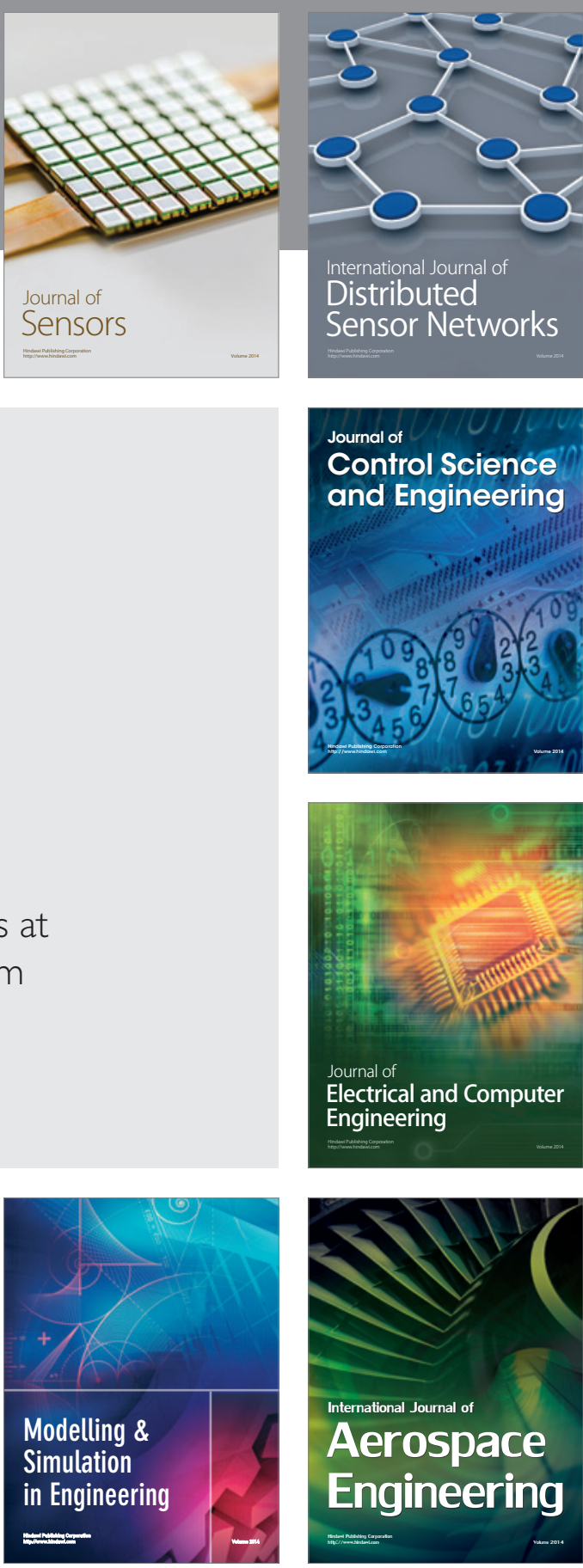

International Journal of

Distributed

Sensor Networks

Journal of

Control Science

and Engineering
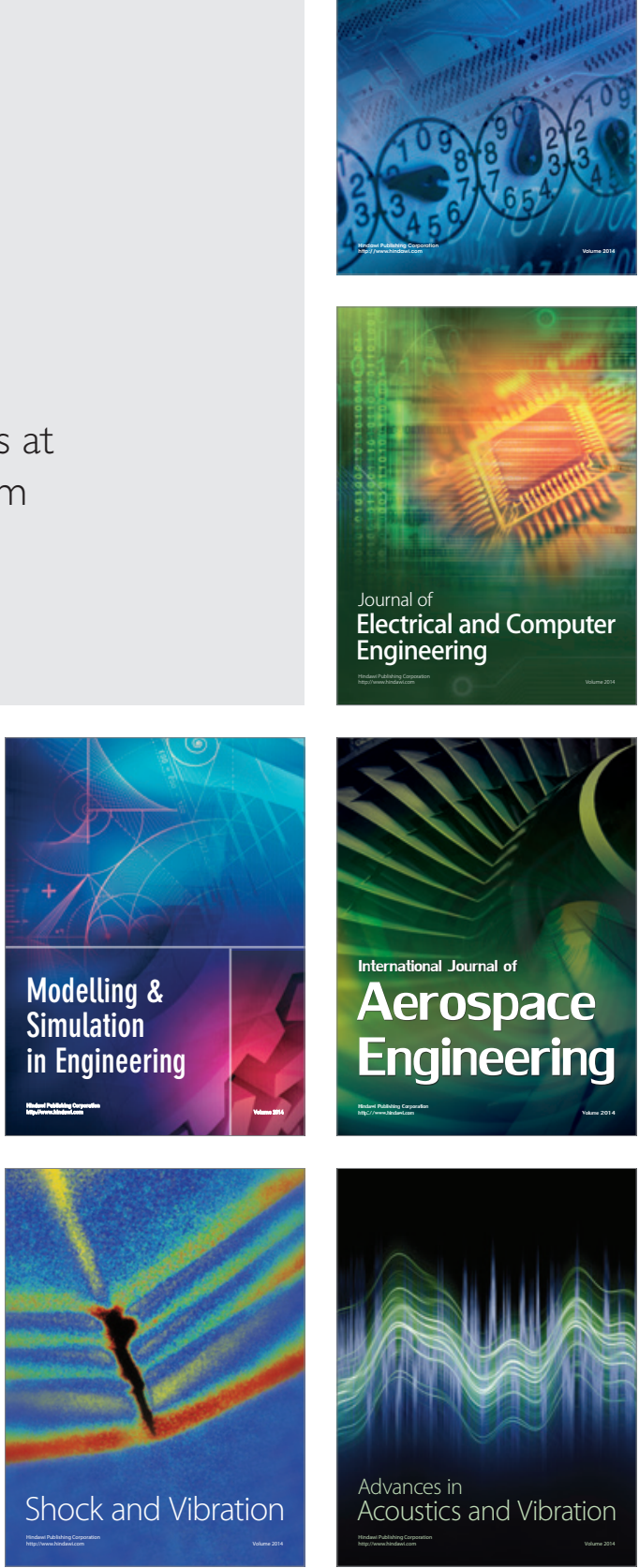